\title{
SsrA-mediated trans-translation plays a role in mRNA quality control by facilitating degradation of truncated mRNAs
}

\author{
YASUFUMI YAMAMOTO, TAKAFUMI SUNOHARA, KAORU JOJIMA, TOSHIFUMI INADA, and HIROJI AIBA \\ Division of Biological Science, Graduate School of Science, Nagoya University, Chikusa, Nagoya 464-8602, Japan
}

\begin{abstract}
An important unsolved question regarding the bacterial SsrA system is the fate of target mRNAs replaced by SsrA RNA during trans-translation. The aim of the present study is to address the potential role of SsrA system in mRNA quality control, focusing on truncated mRNAs that are expected to arise from 3'-to-5' exonucleolytic attack. We found that significant amounts of truncated mRNAs and polypeptides were produced from genes lacking a $\rho$-independent terminator in SsrA-deficient cells. These truncated mRNAs, hence truncated polypeptides, were no longer observed in the presence of SsrA RNA. The data indicate that the SsrA system facilitates degradation of "nonstop" mRNAs by presumably removing the stalled ribosomes. Furthermore, analysis of affinity-purified proteins indicated that truncated polypeptides could be produced even from a gene with an intact $\rho$-independent terminator, although less efficiently, implying that C-terminally truncated proteins and 3'-truncated mRNA may be produced from virtually all protein-coding genes. We conclude that the SsrA system not only promotes the degradation of incomplete polypeptides but also minimizes the synthesis of incomplete polypeptides by facilitating the degradation of truncated $\mathrm{mRNAs}$ that are produced in cells.
\end{abstract}

Keywords: tmRNA; trans-translation; mRNA degradation; quality control; nonstop mRNA

\section{INTRODUCTION}

Several small RNAs with a variety of functions have been identified in bacteria (Storz 2002; Wassarman 2002). Among these, tmRNA, also known as SsrA RNA or $10 \mathrm{Sa}$ RNA, is involved in the unique process referred to as transtranslation (Keiler et al. 1996; Karzai et al. 2000). According to the well-accepted model, when a ribosome stalls on a problematic mRNA, typically at the $3^{\prime}$ end of a truncated mRNA without an in-frame stop codon, SsrA RNA charged with alanine is recruited to the A site of the ribosome together with an associated protein, $\mathrm{SmpB}$, and donates an alanine to the growing polypeptide chain by acting as a tRNA. The ribosome then switches from the mRNA to a short coding sequence in SsrA RNA. Translation continues and terminates normally at the stop codon that follows the SsrA RNA reading frame. The final translation product is a chimeric polypeptide in which the specific 11-residue tag

Reprint requests to: Hiroji Aiba, Division of Biological Science, Graduate School of Science, Nagoya University, Chikusa, Nagoya 464-8602, Japan; e-mail: i45346a@nucc.cc.nagoya-u.ac.jp; fax: 81-52-789-3001.

Article and publication are at http://www.rnajournal.org/cgi/doi/ $10.1261 /$ rna.2174803.
(AANDENYALAA) is attached at the C terminus. The tag sequence targets the product of trans-translation for degradation by ATP-dependent proteases (Keiler et al. 1996; Gottesman et al. 1998; Herman et al. 1998).

There are several situations in which target mRNAs for the SsrA system are produced and the protein tagging occurs. The best-known target for the SsrA system is the $3^{\prime}$ end of a truncated mRNA lacking an in-frame stop codon where the ribosome is expected to stall (Keiler et al. 1996). The truncated "nonstop" mRNA could be generated either by nuclease cleavage of an mRNA or by incomplete transcription. The SsrA-mediated tagging of LacI typically represents this situation because LacI binding to the lac operators could block completion of lac transcription, resulting in truncated lac mRNAs (Abo et al. 2000). A ribosome would also reach the $3^{\prime}$ end of an mRNA when a normal stop codon is erroneously translated either in the presence of nonsense suppressor tRNAs (Ueda et al. 2002) or in the presence of misreading drugs (Abo et al. 2002). The SsrA system appears to act also at a run of rare codons on an mRNA, where ribosomes are expected to stall because of the deficiency of cognate aminoacyl-tRNAs (Roche and Sauer 1999). In addition, recent studies have shown that SsrA 
tagging could occur at a position corresponding to the normal termination codon in certain conditions depending on the presence of rare arginine codons near the adjacent inefficient UGA termination codon (Collier et al. 2002; Hayes et al. 2002b) or the amino acid sequence of the nascent polypeptide prior to stop codons (Hayes et al. 2002a; Sunohara et al. 2002).

The well-established biological roles of the SsrA system are to rescue stalled ribosomes and to target unwanted polypeptides for degradation by cellular proteases (Karzai et al. 2000; Withey and Friedman 2002). On the other hand, little is known about the fate of mRNAs replaced by SsrA RNA during trans-translation. The findings that SsrA RNA is associated with a $3^{\prime}$-to-5' exonuclease (RNase R; Karzai and Sauer 2001) and that RNase E is involved in the maturation of SsrA RNA (Lin-Chao et al. 1999) indicate a link between the SsrA RNA system and RNA metabolism. We found recently that the abundance of an mRNA lacking a stop codon was markedly reduced by the presence of SsrA RNA (Ueda et al. 2002), indicating that a trans-translation process may facilitate the decay of target mRNAs.

The aim of the present study is to address the role of the SsrA system in mRNA metabolism, focusing on degradation intermediates that would be produced by $3^{\prime}$-to- $5^{\prime}$ exonucleolytic digestion of mRNAs. We demonstrate that significant amounts of truncated mRNAs are produced from an mRNA lacking a stem-loop structure at its $3^{\prime}$ end and that these truncated mRNAs could direct the synthesis of heterogeneous truncated polypeptides. Evidence indicates that the truncated mRNAs are recognized by the SsrA system, leading to a rapid degradation of aberrant mRNAs as well as an efficient degradation of SsrA-tagged polypeptides. We conclude that the SsrA system not only promotes the degradation of aberrant polypeptides once they are produced but also minimizes the synthesis of aberrant polypeptides by facilitating the degradation of truncated mRNAs that are continuously produced in cells.

\section{RESULTS}

\section{SsrA RNA does not affect the expression of full-length CRP}

A hairpin structure at the $3^{\prime}$ end of an mRNA often contributes to stabilization of the mRNA by acting as a barrier against 3'-to-5' exonucleolytic attack (Carpousis et al. 1999; Higgins et al. 1993). The Escherichia coli crp gene encoding cAMP receptor protein (CRP) possesses a typical $\rho$-independent terminator, thereby generating an mRNA with a $3^{\prime}$ hairpin structure (Aiba et al. 1982). We showed previously that disruption of the crp terminator leads to a decreased CRP expression as a result of destabilization of the crp mRNA (Aiba et al. 1991; Abe and Aiba 1996). To study the effect of SsrA RNA on mRNA stability, we first examined how SsrA RNA affects the expression of CRP, particularly focusing on the terminatorless crp gene. For this, pHA7M6 and pHA7M9 carrying the crp gene with a defective terminator (Fig. 1) were transformed into $s s r A^{+}$and $s s r A^{-}$strains. The parent plasmid pHA7 carrying the crp gene with a functional $\rho$-independent terminator and a control plasmid pBR322 were also transformed into two strains. Total extracts prepared from cells harboring each of these plasmids were analyzed by Western blotting using anti-CRP antibodies. As expected, the expression of CRP in cells harboring pHA7M6 or pHA7M9 was significantly lower than that in cells harboring pHA7 in an $s s r A^{+}$background, whereas no significant difference in CRP level was observed between $s s r A^{+}$and $s s r A^{-}$cells harboring any one of three pHA7 derivatives (Fig. 2). Therefore, SsrA RNA has little effect on the expression of full-length CRP regardless of the nature of the terminator.

\section{Significant amounts of truncated CRP polypeptides are detected in SsrA-defective cells carrying a terminatorless crp gene}

There is an intrinsic terminator hairpin structure $11 \mathrm{nt}$ downstream of the translation stop codon in the crp gene (Fig. 1). We showed previously that much transcription of crp goes through the terminator and terminates far downstream of the $\operatorname{crp}$ terminator, resulting in longer heterogeneous transcripts when the crp mRNA is normally translated (Abe et al. 1999). The readthrough occurs presumably because the translating ribosome affects RNA polymerase paused at the $\operatorname{crp}$ terminator. The primary heterogeneous transcripts are nibbled back to the stem-loop structure (Abe et al. 1999). The reduced expression of CRP in the terminatorless crp gene is apparently caused by the absence of a barrier against $3^{\prime}$-to- $5^{\prime}$ exonucleolytic attack in the mRNA. When the exonucleolytic digestion enters the coding region beyond the stop codon from the $3^{\prime}$ end on an mRNA being translated, the exonuclease(s) may encounter ribosomes coming from the $5^{\prime}$ end. If this is the case, truncated polypeptides would be produced efficiently from the terminatorless $\mathrm{crp}$ gene.

To examine this possibility, we constructed a plasmid, pYY101, carrying the terminatorless crp gene encoding $\mathrm{His}_{6}$-CRP, which contains a hexahistidine $\left(\mathrm{His}_{6}\right)$ tag at the $\mathrm{N}$ terminus of CRP (Fig. 3A). Another plasmid, pYY100, carrying a variant $c r p$ gene encoding $\mathrm{His}_{6}$-CRP but retaining the intact terminator was also constructed. The $\mathrm{His}_{6}$-CRP was expressed in three different strains with respect to the $s s r A$ allele $\left(s s r A^{-}, s s r A^{+}\right.$, and $\left.s s r A^{D D}\right)$. The $\mathrm{His}_{6}$-CRP proteins were purified by $\mathrm{Ni}^{2+}$-NTA affinity chromatography and analyzed by Western blotting by using either anti-CRP or anti-DD-tag antibodies (Fig. 3B). The anti-CRP-probed Western blots of purified $\mathrm{His}_{6}-\mathrm{CRP}$ proteins from $s s r A^{-}$ cells harboring pYY101 revealed a strong ladder of truncated polypeptides with different intensities along with the full-length $\mathrm{His}_{6}-\mathrm{CRP}$ of $24.4 \mathrm{kD}$ (Fig. 3B, lane 4). These 


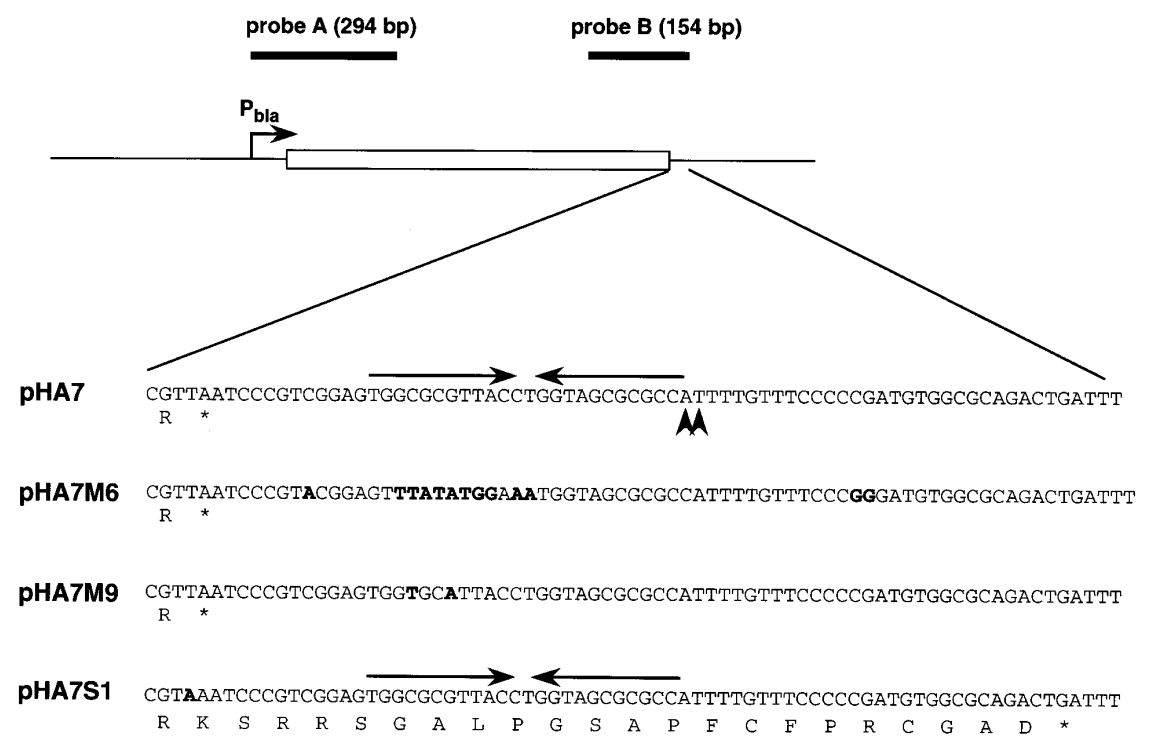

FIGURE 1. Sequences around the wild-type and variant $c r p$ terminators. Plasmid pHA7 carries the $c r p$ gene under the control of the bla promoter, whereas pHA7M6 and pHA7M9 are derivatives of pHA7 in which the terminator stem of $c r p$ is disrupted by base substitutions. Plasmid pHA7S1 is a derivative of pHA7 in which the crp stop codon (TAA) is changed to a sense cordon (AAA). (Upper panel) A diagram of the crp gene in these plasmids. The elevated arrow denotes the start and direction of transcription. The open rectangle represents the coding region for CRP. The black bars represent DIG-labeled DNA probes used for Northern analysis. The figure is not drawn to scale. The nucleotide sequences of the terminator region are shown below the diagram. The GC-rich inverted repeat sequence of the crp terminator is shown by horizontal arrows. The arrowheads indicate major 3' ends of $\operatorname{crp}$ mRNA. The mutational changes are indicated as bold letters. Mutational changes outside of the hairpin structure in pHA7M6 are described previously and are not relevant to the present experiments. Asterisks indicate the translation termination codons. The amino acids encoded by the $3^{\prime}$-flanking region of $\operatorname{crp}$ are given below the nucleotide sequence.

shorter polypeptides should be truncated at their C-terminal side because any fragments missing the $\mathrm{N}$-terminal portion would be excluded by the affinity purification. Most shorter bands were no longer observed when the purified $\mathrm{His}_{6}$-CRP proteins from $s s \mathrm{~A}^{+}$cells harboring pYY101 were analyzed (Fig. 3B, lane 5). This implies that the truncated polypeptides are either eliminated or not produced in the presence of SsrA RNA. To directly detect the SsrA tagging, the purified $\mathrm{His}_{6}-\mathrm{CRP}$ proteins from $s s r A^{D D}$ cells harboring pYY10l were also analyzed by Western blotting. The $s s r A^{D D}$ allele encodes a variant SsrA ${ }^{\mathrm{DD}}$ RNA containing a coding region for a protease-resistant tag in which two alanines at the very $\mathrm{C}$ terminus of the tag sequence have been changed to two aspartates (Roche and Sauer 1999; Abo et al. 2000). The purified $\mathrm{His}_{6}$-CRP proteins from $s s r A^{D D}$ cells harboring pYY101 (Fig. 3B, lane 6) exhibited a ladder of truncated polypeptides that is up-shifted relative to that observed in SsrA-defective cells, indicating that the shorter CRP polypeptides produced from the terminatorless $\mathrm{crp}$ gene were tagged by the SsrA system. In fact, these bands were also recognized with anti-DD-tag antibodies (Fig. 3B, lane 12), indicating that they represent DD-tagged truncated CRP polypeptides. Interestingly, the abundance of tagged bands in $s s r A^{D D}$ cells is far less than that of the truncated bands in $s s r A^{-}$cells (Fig. 3B, lanes 4,6). In addition, analysis of affinity-purified $\mathrm{His}_{6}-\mathrm{CRP}$ proteins indicated that truncated polypeptides are produced, although at a very low level, even from the crp gene with an intact terminator in $s s r A^{-}$ cells (Fig. 3B, lane 1). A faint ladder of DD-tagged polypeptides was detected in the purified proteins from $s s r A^{D D}$ cells harboring pYY100 (Fig. 3B, lanes 3,9). Although the purification of $\mathrm{His}_{6}$-CRP proteins facilitated the detection of heterogeneous CRP polypeptides and their DD-tagging, the truncated polypeptides were also detected when excess amounts of extract $s s A^{-}$cells harboring the terminatorless crp plasmids were analyzed by Western blotting (data not shown).

\section{Degradation intermediates of hairpinless crp mRNA accumulate in SsrA-defective cells}

Bacterial mRNAs are unstable in general, and they are rapidly degraded by a combined action of several endonucle-

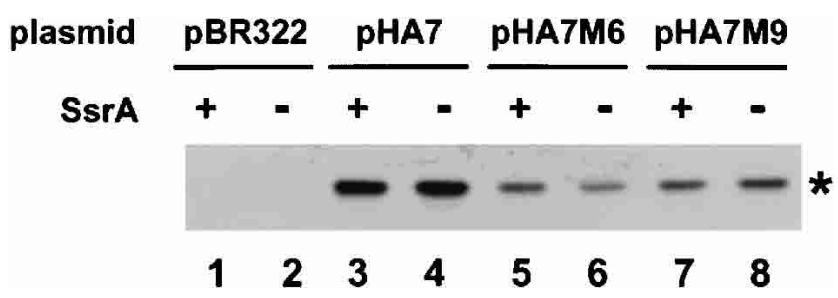

FIGURE 2. Effect of SsrA RNA on the expression of full-length CRP. Total extracts were prepared from PP47 ssr $A^{+}$and PP47 ssr ${ }^{-}$cells harboring pHA7 derivatives. Lysates equivalent to $0.005 \mathrm{OD}_{600}$ units (Miller 1972) were analyzed by Western blotting using anti-CRP antibodies. The band of the full-length CRP $(23.6 \mathrm{kD})$ is shown by an asterisk. 
A
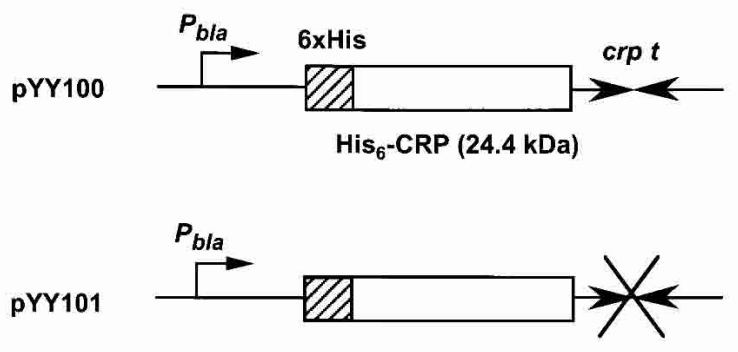

B

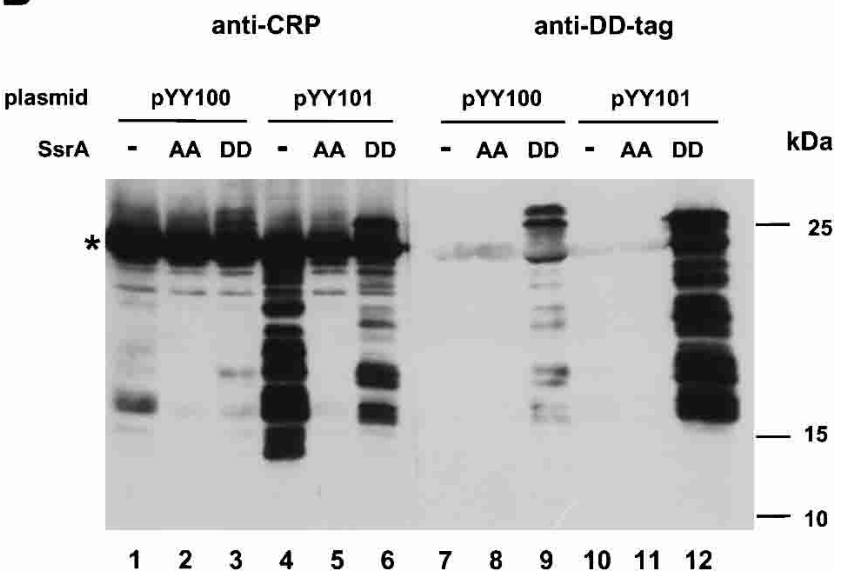

FIGURE 3. Western blot analysis of $\mathrm{His}_{6}$-CRP proteins derived from the wild-type and terminatorless $\mathrm{crp}$ genes. (A) Schematic drawing of the wild-type and terminatorless crp genes encoding $\mathrm{His}_{6}$-CRP in pYY100 and pYY101. The elevated arrow denotes the start and direction of transcription. The hatched and open rectangles represent the coding regions for $6 \times$ His and CRP, respectively. The figure is not drawn to scale. The $\rho$-independent $c r p$ terminator is shown by horizontal arrows. $(B)$ Western blot analysis of $\mathrm{His}_{6}$-CRP proteins. The $\mathrm{His}_{6}$-CRP proteins were expressed in three different strains, TA331 $\left(s s r A^{-}\right)$, W3110 $\left(s s r A^{+}\right)$, and TA371 $\left(s s r A^{D D}\right)$, harboring pYY100 and pYY101. W3110 $\left(s s r A^{+}\right)$and TA371 $\left(s s r A^{D D}\right)$ express the wild-type SsrA RNA (AA) and SsrA ${ }^{\mathrm{DD}}$ RNA (DD), respectively. The proteins were purified by $\mathrm{Ni}^{2+}$-NTA affinity chromatography. The purified proteins of $1 \mu \mathrm{g}$ were subjected to Western blot analysis using antiCRP antibodies (lanes 1-6) and anti-DD-tag antibodies (lanes 7-12). The position of the full-length $\mathrm{His}_{6}$ CRP $(24.4 \mathrm{kD})$ is shown by an asterisk. Size standards are on the right.

ases and exonucleases in Escherichia coli cells (Carpousis et al. 1999; Coburn and Mackie 1999; Regnier and Arraiano 2000; Steege 2000). Although RNase E is believed to be the major endonuclease, two $3^{\prime}$-to- $5^{\prime}$ exonucleases, polynucleotide phosphorylase (PNPase) and ribonuclease II (RNase II), are predominantly responsible for the exonucleolytic degradation of mRNA. If $3^{\prime}$-to-5' exonucleolytic attack occurs prior to endonuclease attack in the $5^{\prime}$ region, mRNA fragments truncated at the $3^{\prime}$ side would be produced. However, it is difficult to detect such degradation intermediates because of their instability except when the mRNA has a hairpin structure at the $3^{\prime}$ end that could stabilize mRNA by acting as a barrier against $3^{\prime}$-to- $5^{\prime}$ exonucleolytic attack.
The production of truncated CRPs and their tagging by the SsrA system can be explained either by generation of truncated mRNAs or by stalling of ribosomes at rare codons. To test whether truncated crp mRNAs are generated, total RNAs from the wild-type and terminatorless crp gene were analyzed by Northern blotting in three genetic backgrounds with respect to the $s s r A$ allele. We first used a DNA probe specific to the $5^{\prime}$ portion of the $\operatorname{crp}$ mRNA. The crp mRNA expressed from the wild-type $\operatorname{crp}$ gene on pHA7 gave a major band of $\sim 700 \mathrm{nt}$ in all three strains (Fig. 4A, lanes 2-4). This band represents a mature $c r p$ mRNA that is generated from the longer primary transcripts by RNA processing, and its $3^{\prime}$ end was mapped at the distal portion of the terminator hairpin (Abe et al. 1999; Ueda et al. 2002). In addition, longer dispersed bands were also observed along with a faint smear of shorter RNAs. The longer bands are produced by transcriptional readthrough at the crp terminator, whereas the shorter smear may represent a variety of degradation intermediates of $c r p$ mRNA. In any case, SsrA RNA did not affect the pattern of Northern bands when the crp terminator was intact. When the $\rho$-independent terminator was disrupted, the major crp mRNA band was no longer observed in the case of pHA7M6 (Fig. 4A, lanes 5-7) or was markedly decreased in the case of pHAM9 (Fig. 4A, lanes 8-10), whereas the longer dispersed bands increased in both $s s r A^{+}$and $s s r A^{-}$cells because of increased transcriptional readthrough.

A striking observation regarding the effect of disruption of the $\rho$-independent terminator, however, was the appearance of an additional intensive ladder/smear of truncated RNAs ranging in size from 300 to $650 \mathrm{nt}$ in $s s r A^{-}$cells (Fig. $4 \mathrm{~A}$, lanes 5,8$)$. These bands were no longer observed in $s s r A^{+}$and $s s r A^{D D}$ cells (Fig. 4A, lanes 6,7,9,10), indicating that the truncated RNAs are rapidly degraded in the presence of either SsrA RNA or SsrA ${ }^{\mathrm{DD}}$ RNA. The shorter RNAs are expected to be derived from the $5^{\prime}$ portion of the $\mathrm{crp}$ mRNA because a $5^{\prime}$-specific probe was used in the Northern analysis. To confirm this, Northern blot analysis was also carried out by using a DNA probe specific to the $3^{\prime}$ portion of crp mRNA. As shown in Figure 4B, the ladder of shorter RNAs was no longer observed, but the mature, longer crp mRNAs were detected as in the case of the $5^{\prime}$ probe. We conclude that the truncation in shorter RNAs primarily arises from the $3^{\prime}$ end of $\operatorname{crp}$ mRNA by the action of $3^{\prime}$-to-5' exonucleases. Our experiments apparently rule out an alternative possibility that stalling of the ribosome per se is a source of mRNA cleavage, because few truncated RNAs were generated from the normal crp mRNA possessing the terminator hairpin (Fig. 4A, lane 2).

\section{SsrA RNA facilitates degradation of nonstop mRNA}

The Northern blot analysis shown in Figure 4 strongly indicates that SsrA RNA facilitates the degradation of truncated mRNAs derived from the terminatorless crp gene. 
However, it was difficult to examine the effect of SsrA RNA on the stability of these truncated mRNAs directly because they were dispersed and presumably too unstable to be detected in $s s A^{+}$cells. To determine whether SsrA-RNAmediated trans-translation influences the stability of mRNAs lacking a stop codon, we focused on a discrete nonstop crp mRNA derived from pHA7S1 carrying a variant crp gene in which the normal TAA stop codon of CRP was replaced with an AAA codon (Fig. 1). We found previously that the abundance of this nonstop $\operatorname{crp}$ mRNA derived from $\mathrm{pHA7S1}$ was markedly reduced but still detectable in the presence of SsrA RNA (Ueda et al. 2002). This observation was confirmed again in this study as shown in Figure 5A. The reduction of the nonstop crp mRNA in the presence of SsrA RNA (Fig. 5A, lanes 3,4) strongly indicates that it is somehow destabilized by SsrA RNA. To confirm this, pHA7S1 and pHA7 were transformed in $s s r A^{-}$and $s s r A^{+}$strains. Cells were grown to exponential phase, and rifamipicin was added to prevent further initiation of transcription. Cellular RNAs were isolated at various times after the addition of rifampicin and subjected to Northern blot analysis (Fig. 5B). The chemical half-life of the normal crp mRNA derived from pHA7 was $2.5 \mathrm{~min}$ in $s s r A^{-}$cells and 2.3 min in $s s r A^{+}$cells. Thus, SsrA RNA had a small effect on the stability of the normal $\operatorname{crp}$ mRNA. On the other hand, the half-life of crp mRNA lacking a stop codon was dramatically decreased to $0.6 \mathrm{~min}$ in $s s r A^{+}$cells, whereas it was also $2.5 \mathrm{~min}$ in $s s r A^{-}$cells. The simplest interpretation of these results is that the nonstop crp mRNA derived from pHA7S1 is stabilized in the absence of SsrA RNA by the stalled ribosomes at the $3^{\prime}$ end and that SsrA-RNA-mediated trans-translation facilitates degradation of the nonstop mRNA by releasing the stalled ribosome. It is reasonable to conclude that SsrA RNA also facilitates the degradation of heterogeneous truncated nonstop mRNAs derived from the terminatorless crp gene.

\section{SsrA-mediated tagging and degradation of truncated polypeptides derived from genes lacking a terminator hairpin}

So far, we have shown that the disruption of $\operatorname{crp}$ terminator leads to the production of significant amounts of 3 '-truncated mRNAs and C-terminally truncated CRP polypeptides. The SsrA system facilitates the rapid degradation of these mRNAs in addition to efficient tagging and degradation of the incomplete polypeptides. Although many mRNAs possess a hairpin structure at their $3^{\prime}$ ends primarily because of the presence of a $\rho$-independent terminator, others intrinsically lack a 3 '-hairpin structure. It is certainly interesting to examine whether truncated mRNAs, thereby truncated polypeptides, are produced from such naturally hairpinless genes. For this, we analyzed the expression of $m e l R$ and soxR genes. The melR gene encodes a transcription activator, MelR, which is essential for induction of the $m e l A B$ operon responsible for melibiose metabolism (Webster et al. 1987), whereas the soxR gene encodes a transcriptional regulator, SoxR, that is involved in the regulation of a superoxide stress regulon (Amabile-Cuevas and Demple 1991). Both the melR and soxR genes are monocistronic, and there is no clear stem-loop structure in their $3^{\prime}$-flanking region. The coding regions of these genes with their 200-bp 3'-flanking region were cloned in a pQE-80L vector in which $\mathrm{His}_{6}$-tagged proteins can be expressed under the control of the isopropyl-thiogalactoside (IPTG)-inducible promoter. The resulting plasmids, pMelR and pSoxR, were introduced into three different strains with respect to the ssr A allele. Cells were grown to a mid-log phase, and the expression of $\mathrm{N}$-terminally $\mathrm{His}_{6}$-tagged proteins was induced by adding IPTG. Total extracts were analyzed by Western blotting using a monoclonal antibody to the histidine tag. Several truncated polypeptides were detected along with full-length $\mathrm{His}_{6}$-tagged proteins in $s s r A^{-}$cells (Fig. 6A,B, lane 1), but they were no longer observed in

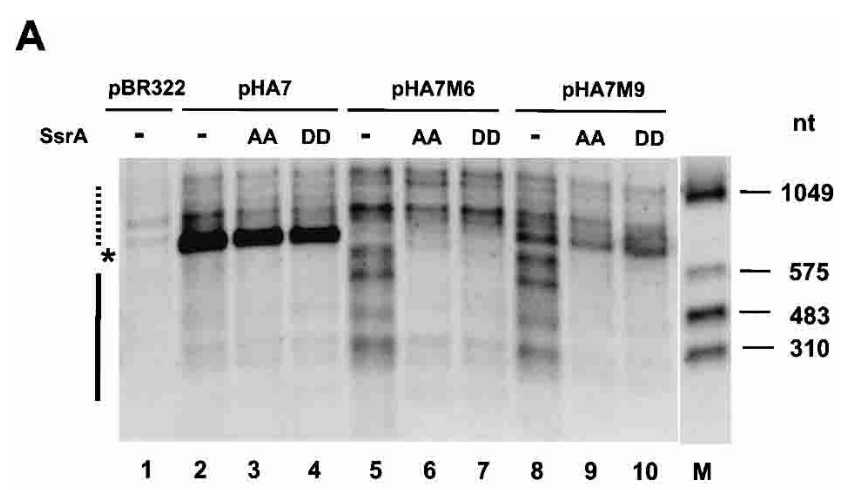

B

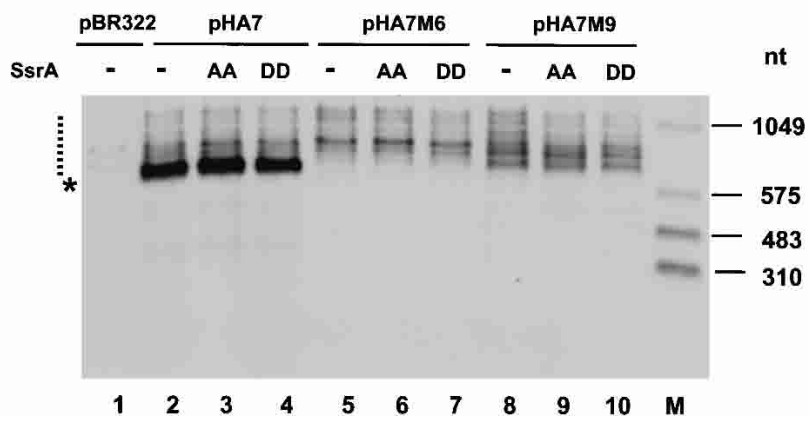

FIGURE 4. Northern blot analysis of mRNAs derived from the wildtype and terminatorless $\mathrm{crp}$ genes. The $\mathrm{crp}$ mRNAs were expressed in three different cells, TA331 $\left(s s r A^{-}\right)$, W3110 $\left(s s r A^{+}\right)$, and TA371 $\left(s s r A^{D D}\right)$, harboring pHA7 derivatives. Total RNAs $(5 \mu \mathrm{g})$ were subjected to Northern blot analysis using a DIG-labeled $5^{\prime}$ probe A $(A)$ and $3^{\prime}$ probe $\mathrm{B}(B)$. Asterisks indicate the major crp mRNA band whose $3^{\prime}$ ends are mapped just after the stem and loop structure of the crp terminator. The vertical bar and the dashed vertical bar represent truncated $c r p$ mRNAs and longer dispersed $c r p$ mRNAs, respectively. The positions of RNA size markers are shown on the right. 
A

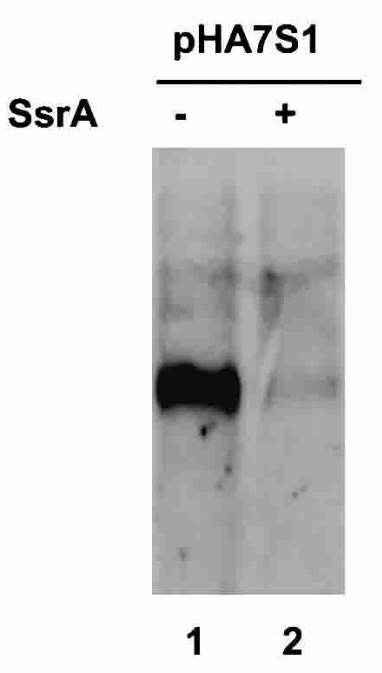

B

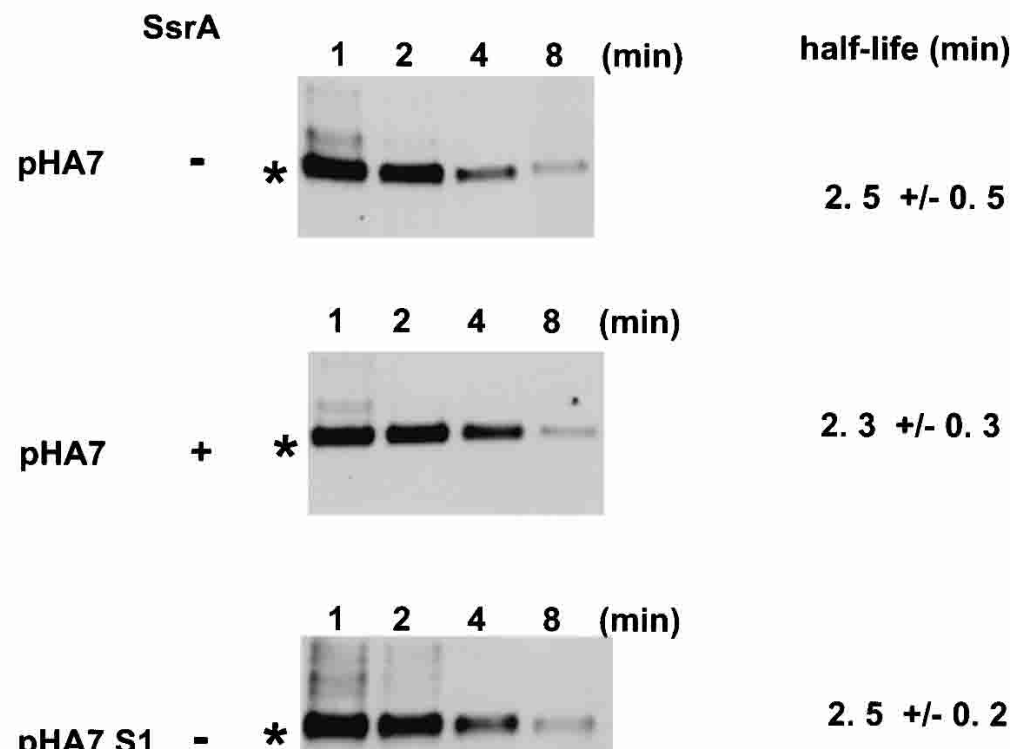

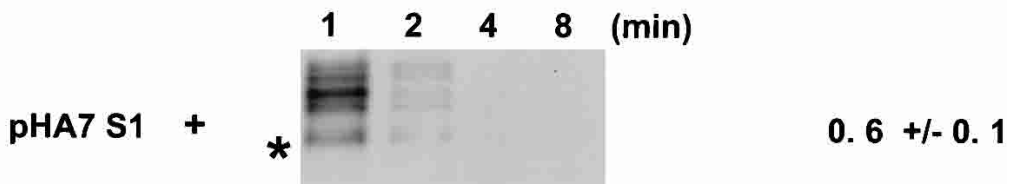

FIGURE 5. Effect of SsrA RNA on the stability of normal and nonstop crp mRNAs. (A) Northern blot analysis of $\operatorname{crp}$ mRNAs derived from the pHA7 and pHA7S1. The $\operatorname{crp}$ mRNAs were expressed in TA331 $\left(s s r A^{-}\right)$and W3110 $\left(s s r A^{+}\right)$harboring pHA7 or pHA7S1. Total RNAs $(5 \mu \mathrm{g})$ were subjected to Northern blot analysis using a DIG-labeled 5' probe A. The major $\operatorname{crp}$ mRNA band is shown by an asterisk. (B) Northern blot analysis of $\operatorname{crp}$ mRNAs prepared from rifampicin-treated cells. W3110 $\left(s s r A^{+}\right)$and TA331 $\left(s s r A^{-}\right)$harboring pHA7 or pHA7S1 were grown to OD $600=0.8$. Rifampicin $(200 \mu \mathrm{g} / \mathrm{mL})$ was added to prevent further initiation of transcription. Cellular RNAs were prepared at indicated times after the addition of rifampicin. Total RNAs $(10 \mu \mathrm{g})$ were subjected to Northern blot analysis. The bands corresponding to the major $\mathrm{crp}$ mRNA indicated by asterisks were quantified using Bioimage Analyzer LAS-1000 (Fuii). Half-lives were determined by plotting the percent of mRNA remaining versus time on a semilog plot. The values are the average of three independent experiments.

$s s r \mathrm{~A}^{+}$cells (Fig. 6A,B, lane 2), indicating that they are efficiently eliminated by the SsrA system. When the $\mathrm{His}_{6}$-proteins were expressed in $s s r A^{D D}$ cells, truncated peptides with different mobilities were detected (Fig. 6A,B, lane 3). We confirmed by Western blot analysis using anti-DD-tag antibodies that these bands are indeed DD-tagged truncated peptides (data not shown). It should be noted again that the abundance of the DD-tagged polypeptides was markedly reduced relative to that of the truncated polypeptides produced in $s s r A^{-}$cells (Fig. 6A,B, lanes 1,3).

\section{Detection of truncated $m R N A s$ derived from genes lacking a terminator hairpin}

To test whether truncated melR and soxR mRNAs are generated, three different strains with respect to the $s s r A$ allele harboring pMelR or pSoxR were grown to a mid-log phase, and the RNA expression was induced by adding IPTG. Then, total RNAs were prepared and analyzed by Northern blotting using DNA probes specific to the $5^{\prime}$ portion of $m e l R$ and soxR mRNAs. In the case of melR, accumulation of short truncated RNAs ranging in size from 1050 to $250 \mathrm{nt}$ was clearly observed in $s s r A^{-}$cells (Fig. 7, lane 1) along with a longer RNA band of $\sim 1300 \mathrm{nt}$. The truncated RNAs were dramatically reduced in either $s s r A^{+}$or $s s r A^{D D}$ cells, whereas the abundance of the longer band was not affected by SsrA RNA (Fig. 7, lanes 2,3). The longer discrete band may be produced because significant portions of the melR transcription reach the vector-derived $\rho$-independent $\lambda$ t0 transcription terminator located $\sim 250$ bp downstream of the melR stop codon. Similarly, short, truncated RNAs ranging in size from 550 to $250 \mathrm{nt}$ were clearly observed in $s s r A^{-}$ cells along with a longer RNA band of $\sim 800 \mathrm{nt}$ when mRNAs produced from the cloned soxR gene were analyzed by Northern blotting (Fig. 7, lane 4). Again, the truncated RNAs but not the longer RNA were markedly reduced in either the $s s r A^{+}$or the $s s r A^{D D}$ strain (Fig. 7, lanes 5,6). These results are consistent with a view that the truncation in 
A

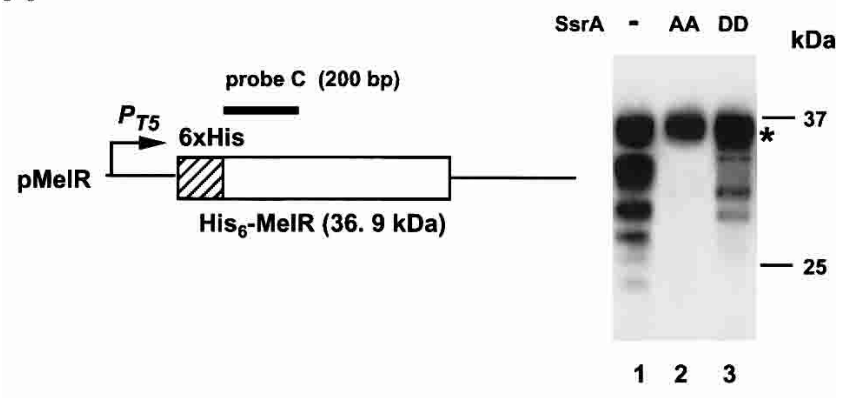

B
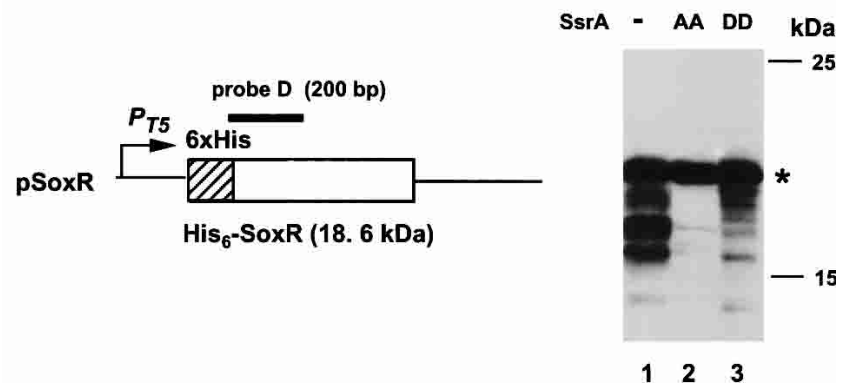

FIGURE 6. Western blot analysis of proteins derived from the cloned melR $(A)$ and soxR (B) genes. (Left panels) Schematic drawings of variant melR and soxR genes encoding $\mathrm{N}$-terminally $\mathrm{His}_{6}$-tagged proteins. The elevated arrow denotes the start and direction of transcription. The hatched and open rectangles represent the coding regions for $6 \times$ His and MelR or SoxR, respectively. The figures are not drawn to scale. The black bars represent the DIG-labeled DNA probes used for Northern analysis. (Right panels) The results of Western blot analysis of N-terminally $\mathrm{His}_{6}$-tagged proteins. TA331 $\left(s s r A^{-}\right)$, W3110 $\left(s s r A^{+}\right)$, and TA371 $\left(s s r A^{D D}\right)$, harboring pMelR or pSoxR, were grown to $\mathrm{OD}_{600}=0.7$. The synthesis of $\mathrm{His}_{6}$-tagged proteins was induced for 20 min by adding $0.1 \mathrm{mM}$ IPTG. Total extracts were prepared and lysates equivalent to $0.1 \mathrm{OD}_{600}$ unit were subjected to Western blot analysis using anti-His-tag antibody. Asterisks indicate the positions of the full-length $\mathrm{His}_{6}$-MelR $(36.9 \mathrm{kD})$ or $\mathrm{His}_{6}$-SoxR $(18.6 \mathrm{kD})$. Size standards are on the right.

shorter RNAs arises from the action of $3^{\prime}$-to-5' exonucleases and that stalled ribosomes at the $3^{\prime}$ end of truncated mRNAs stabilize the mRNAs in the absence of SsrA RNA. The transcription termination in genes lacking a $\rho$-independent terminator may occur over a wide region downstream of the gene, leading to heterogeneous primary transcripts that are subsequently subjected to 3 '-to-5' exonuclease degradation, resulting in truncated RNAs. The translating ribosomes may block the action of exonucleases to stabilize these shorter RNAs. The stalled ribosomes would be released from the mRNAs in the presence of SsrA RNA, leading to destabilization of the truncated mRNAs.

\section{DISCUSSION}

In this paper, we addressed the role of the SsrA system in mRNA quality control by focusing on the fate of truncated
mRNAs that are expected to arise from $3^{\prime}$-to- $5^{\prime}$ exonucleolytic attack. An important finding in the present work is that significant amounts of truncated crp mRNAs accumulate in cells lacking SsrA RNA when the $\rho$-independent terminator of the $\operatorname{crp}$ gene is disrupted. These truncated mRNAs, which are presumably degradation intermediates of $3^{\prime}$-to- $5^{\prime}$ exonucleolytic attack, were detected by a $5^{\prime}$ probe but not by a $3^{\prime}$ probe (Fig. 4A,B), and they are able to direct the synthesis of truncated polypeptide (Fig. 3). The truncated mRNAs and polypeptides are no longer observed in the presence of SsrA RNA (Figs. 3, 4). In addition, we found that significant amounts of truncated mRNAs and polypeptides can be generated from several cloned genes like melR and soxR intrinsically lacking a $\rho$-independent terminator in the absence of SsrA RNA, as in the case of the terminatorless crp gene (Figs. 6, 7). This implies that various truncated mRNAs are continuously generated from many genes lacking a $3^{\prime}$ secondary structure in cells. Furthermore, analysis of affinity-purified $\mathrm{His}_{6}$-CRP proteins indicated that truncated polypeptides could be produced, although less efficiently, even from a gene with an intact $\rho$-independent terminator (Fig. 3). This strongly indicates that C-terminally truncated proteins and $3^{\prime}$-truncated mRNA may be produced from all protein-coding genes to various extents. It is apparent that the SsrA system must deal extensively with these aberrant mRNAs and polypeptides.

Why do the truncated mRNAs and truncated polypeptides accumulate in the absence of SsrA RNA? A possible explanation would be that SsrA RNA somehow prevents the exonucleolytic digestion of the longer transcripts. However, this is unlikely because SsrA RNA does not affect the level of the longer transcripts. Another possibility would be that transcription elongation is somehow prevented in the absence of SsrA RNA. This is also unlikely because the deficiency of SsrA RNA does not affect expression of the fulllength CRP (Fig. 2). The simplest interpretation of the SsrA effect is that SsrA RNA facilitates the degradation of truncated mRNAs lacking a stop codon. Indeed, we showed that the stability of a specific nonstop crp mRNA generated from a variant crp gene in which the normal stop codon is changed to a sense codon is dramatically reduced in the presence of SsrA RNA (Fig. 5). This is consistent with a view that the truncated mRNAs are rapidly eliminated in the presence of SsrA RNA, but they are stabilized in the absence of SsrA RNA. The exonucleases would attack efficiently the $3^{\prime}$ end of any mRNAs without a stem-loop structure (Fig. $8 \mathrm{~A})$. When the exonuclease enters the coding region beyond the stop codon, it would encounter a ribosome coming from the $5^{\prime}$ end. In this situation, the exonucleolytic digestion would be transiently blocked by the ribosome stalled at the $3^{\prime}$ end of the truncated mRNA when SsrA RNA is not available (Fig. $8 \mathrm{~B}$ ). This is why the truncated mRNAs can be detected by Northern analysis. The fact that the truncated mRNAs and polypeptides exhibit a ladder of discrete bands rather than a smear indicates that the movement of ribo- 


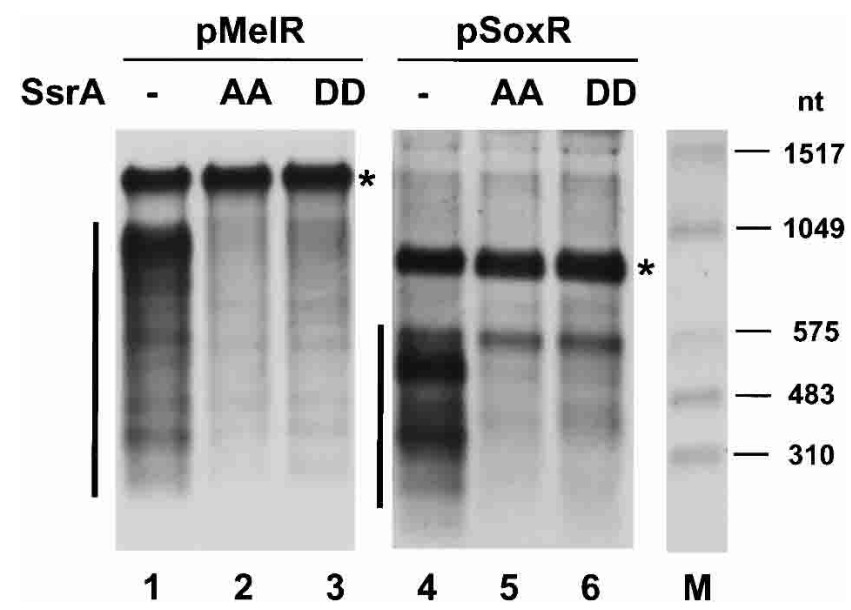

FIGURE 7. Northern blot analysis of mRNAs derived from naturally terminatorless genes. TA331 $\left(s s r A^{-}\right)$, W3110 $\left(s s A^{+}\right)$, and TA371 $\left(s s r A^{D D}\right)$, harboring pMelR or pSoxR, were grown to $\mathrm{OD}_{600}=0.7$. The synthesis of mRNAs was induced for $20 \mathrm{~min}$ by adding $0.1 \mathrm{mM}$ IPTG. Total RNAs $(5 \mu \mathrm{g})$ were subjected to Northern blot analysis using a DIG-labeled probe specific to the $5^{\prime}$ portion of melR or soxR mRNA. Asterisks indicate the extended mRNAs that reached the $\lambda$ t0 terminator derived from the vector. The vertical bars represent the truncated mRNAs. The sizes of the melR- and soxR-mRNA coding regions are $\sim 1050$ and $\sim 550 \mathrm{nt}$, respectively. The positions of RNA size markers are shown on the right.

somes and/or exonucleases on mRNA is not uniform. The stabilization of truncated mRNAs may allow repeated translation of these mRNAs whenever their $5^{\prime}$ portions are functional for the entry of a new ribosome. This leads to the accumulation of significant amounts of truncated polypeptide. On the other hand, the stalled ribosome would be released from the $3^{\prime}$ end in the presence of wild-type SsrA RNA or SsrA ${ }^{\mathrm{DD}}$ RNA and then the exonuclease would chew back to the next ribosome (Fig. 8C). SsrA RNA would again come in and remove the next ribosome followed by exonuclease. This scenario would be repeated until RNA is degraded. SsrA RNA could also recruit exonucleases to the $3^{\prime}$ end of the mRNA in addition to releasing the stalled ribosome. In any case, it is clear that SsrA RNA facilitates degradation of truncated mRNAs by exonucleases. The rapid elimination of truncated mRNAs should prevent further production of truncated polypeptides. This explains very well why the abundance of DD-tagged short polypeptides was far less than that of untagged truncated polypeptides, because only the truncated polypeptide produced by the first round of translation may be tagged by the SsrA system. We conclude that the SsrA system acts as an excellent dual quality-assurance mechanism by facilitating degradation of aberrant polypeptides once produced and by preventing further production of aberrant polypeptides through rapid degradation of aberrant mRNAs.

Although PNPase and RNase II are two major $3^{\prime}$-to-5' exonucleases (Coburn and Mackie 1999; Regnier and Arraiano 2000; Steege 2000), the recent finding that SsrA RNA is associated with RNase R, another $3^{\prime}$-to-5' exonuclease
(Karzai and Sauer 2001), raised a possibility that this endonuclease could also play a role in the degradation of truncated mRNAs. However, disruption of the rnr gene encoding RNase R had no effect on the SsrA-mediated acceleration of mRNA decay, indicating that RNase $\mathrm{R}$ does not play a significant role in the SsrA-mediated rapid degradation of truncated mRNAs (data not shown).

The tagging and degradation of aberrant polypeptides is a hallmark feature of the SsrA-mediated quality control system. However, recent studies have indicated that the primary biological role is the release of stalled ribosomes from mRNAs rather than the destruction of unwanted proteins, because a mutant $s s r A$ gene encoding a protease-resistant tag sequence could rescue several phenotypes of SsrA-defective cells (Withey and Friedman 1999; Huang et al. 2000; Abo et al. 2002). The SsrA-mediated ribosome release has been proposed to have the dual effects of allowing upstream ribosomes to continue translation and returning the stalled ribosomes to the available pool (Withey and Friedman 2002). The present study strongly indicates that an additional important role of the ribosome release would be to facilitate degradation of aberrant mRNAs, hence to prevent the synthesis of aberrant polypeptides.

The nature of SsrA RNA as an mRNA was discovered through fine analyses of a series of truncated proteins accumulated in inclusion bodies when the cDNA of murine interleukin-6 (IL-6) was expressed in E. coli cells (Tu et al. 1995). These proteins varied in size but retained the same 11-amino-acid C-terminal tag that originates from the ssr A gene of E. coli. It was also shown that a significant portion of IL-6 mRNA transcripts were truncated at the $3^{\prime}$ end. This appeared to account for the progressive truncation of IL-6 from its $\mathrm{C}$ terminus. Although this study along with another finding on the tRNA nature of SsrA (Komine et al. 1994; Ushida et al. 1994) led to the trans-translation model, it had been unclear how these truncated mRNAs were generated. The present study has given a possible answer to this question. We believe that the truncated IL-6 proteins were produced from truncated IL-6 mRNAs that were presumably generated by exonucleolytic digestions caused by the lack of $3^{\prime}$ secondary structure in the IL-6 mRNA as observed in several genes described in this work.

The SsrA-mediated degradation of truncated mRNAs is analogous to an mRNA surveillance mechanism found recently in eukaryote cells that eliminates transcripts lacking termination codons (Frischmeyer et al. 2002; Maquat 2002a; van Hoof et al. 2002). However, the bacterial nonstop mRNA decay has several different features from those of the eukaryotic counterpart. First, the recognition and the release of the stalled ribosome are mediated by SsrA RNA along with $\mathrm{SmpB}$ protein in bacteria, whereas they are mediated by several protein factors including the exosome complex in eukaryotes. It would be an interesting question whether a tRNA-like RNA is also involved in the recognition and the release of the stalled ribosome in eukaryotes. 
A

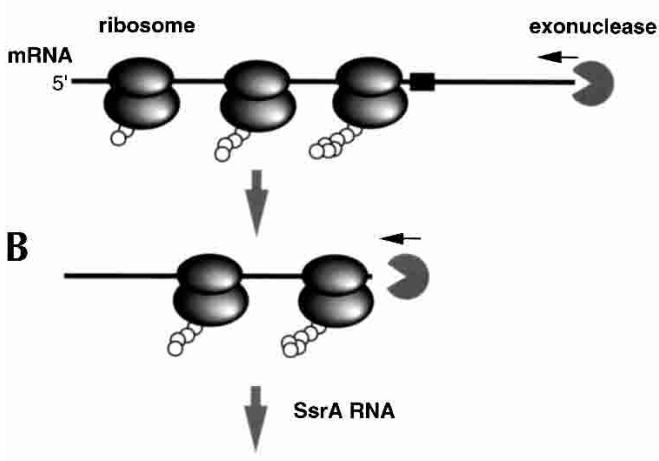

C

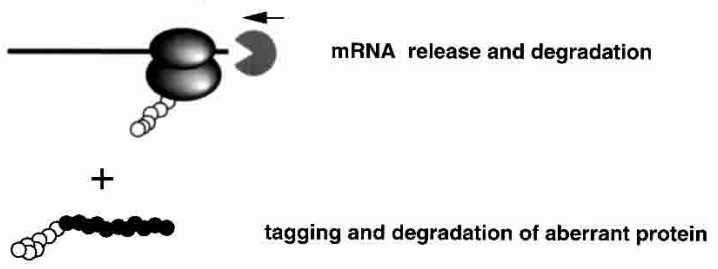

tagged protein

$+$

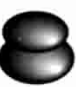

released ribosome

recycling of ribosome

FIGURE 8. Model for mRNA quality control by SsrA RNA. (A) The $3^{\prime}$ end of an mRNA is attacked by $3^{\prime}$-to- $5^{\prime}$ exonucleases (RNase II and/or PNPase). When a ribosome translates the entire coding region before an exonuclease reaches the stop cordon (shown by a closed box), the full-length polypeptide is normally produced. (B) When the exonuclease goes through the stop codon, it encounters another ribosome on the mRNA. The mRNA is protected from the attack of the exonuclease by the stalled ribosome in the absence of SsrA RNA. $(C)$ When the SsrA system is functional, the mRNA is released from the stalled ribosome as part of the trans-translation process, and then the exonuclease chews back the truncated mRNA to the next ribosome. Then SsrA RNA acts to remove the next ribosome followed by exonuclease. This scenario is repeated until the truncated mRNA is degraded. Thus, SsrA RNA contributes to mRNA quality control by facilitating degradation of truncated mRNA. The rapid degradation of truncated mRNA prevents the synthesis of truncated proteins. SsrA RNA-mediated trans-translation is acting as a multiple quality-control system in bacterial cells.

Second, the aberrant polypeptides translated from nonstop mRNAs are tagged and degraded by the SsrA system in bacteria, whereas no mechanism regarding the degradation of protein products of nonstop mRNAs is known so far in eukaryotes. Another well-studied mRNA quality-assurance mechanism in eukaryote cells is nonsense-mediated mRNA decay (NMD), in which mRNAs harboring premature termination codons are preferentially eliminated by coordinated actions of surveillance factors including $5^{\prime}$-to- $3^{\prime}$ exonucleases (Maquat 2002b). On the other hand, it is known that halting translation within an early gene of a polycistronic operon by nonsense mutation causes a significant reduction in the expression of distal genes in bacteria (Adhya and Gottesman 1978). This phenomenon, referred

to as polarity, is believed to occur because RNA polymerase may encounter a previously latent $\rho$-dependent transcription terminator that becomes active because of the lack of translation. It remains to be studied how nonsense mutation affects mRNA stability in bacteria and whether bacterial cells also have an mRNA surveillance mechanism analogous to NMD.

\section{MATERIALS AND METHODS}

\section{Media and growth conditions}

Cells were grown aerobically at $37^{\circ} \mathrm{C}$ in Luria-Bertani (LB) medium (Miller 1972). Antibiotics were used at the following concentrations: ampicillin $(50 \mu \mathrm{g} / \mathrm{mL})$, kanamycin $(50 \mu \mathrm{g} / \mathrm{mL})$, and chloramphenicol $(30 \mu \mathrm{g} / \mathrm{mL})$. Bacterial growth was monitored by determining the optical density at $600 \mathrm{~nm}$.

\section{Strains and plasmids}

The E. coli K-12 strains and plasmids used in this study are listed in Table 1. Strains YA002 and YA004, PP47 derivatives in which the $r n r$ gene was deleted, were constructed by using the gene knockout system of Datsenko and Wanner (2000). Plasmid pHA7M9, a derivative of pHA7 in which the crp terminator was mutated by a 2-base substitution in the hairpin region, was constructed by PCR mutagenesis. Plasmid pYY100 was constructed as follows. A $6 \times$ His coding sequence was introduced at the position just after the first Met residue of the crp ORF by PCR mutagenesis with primers 5'-TAAAGCTTAAGGAGGTTGTATGCATCAT CATCATCATCATGGCAAACCGCAAACAGACCC-3' (cro-crp-1 primer) and 5'-GTATCACGAGGCCCTT-3' (pBR-RI primer) using pHA7 as a template. The amplified DNA fragment was digested with HindIII and EcoRI, and cloned into the HindIII- and EcoRI-digested pBR322 plasmid. The resulting plasmid, pYY100, contains a modified $c r p$ gene encoding $\mathrm{His}_{6}$-CRP transcribed from the bla promoter of pBR322, and has $\lambda$ cro SD sequence. Similarly, plasmid pYY101 carrying a modified terminatorless crp gene encoding $\mathrm{His}_{6}$-CRP was constructed using pHA7M9 as a PCR template. Plasmid pMelR was constructed as follows. The coding sequence for melR missing the first Met residue was amplified by PCR using primers 5'-AATGGATCCAATACAGATACGTTTAT GTGCAGC-3' (B-melR) and 5'-AATAAGCTTATCATTGCTTAC CCGGTTA-3' (H-melR). The amplified fragment was digested with BamHI and HindIII, and cloned into the HindIII- and EcoRIdigested pQE-80L. The resulting plasmid, pMelR, contains a modified melR gene encoding $\mathrm{His}_{6}$-MelR under the control of an IPTG-inducible T5 promoter. Similarly, plasmid pSoxR expressing His $_{6}$-SoxR was constructed using the following primers: $5^{\prime}-\mathrm{AC}$ GCGGATCCGAAAAGAAATTACCCCGCATTAAG-3' (B-soxR) and 5 '-ACCCAAGCTTAAAATATTGCGGTTAAAAAGTATAC$3^{\prime}(\mathrm{H}-\mathrm{soxR})$.

\section{Western blotting}

Bacterial cells harboring pHA7 derivatives were grown in LB medium containing appropriate antibiotic(s) to mid-log phase. Culture samples $(1 \mathrm{~mL})$ were centrifuged, and the pellets were sus- 
TABLE 1. Bacterial strains and plasmids used in this study

\begin{tabular}{|c|c|c|}
\hline $\begin{array}{l}\text { Strain/ } \\
\text { plasmid }\end{array}$ & $\begin{array}{l}\text { Relevant genotype } \\
\text { and property }\end{array}$ & Source \\
\hline \multicolumn{3}{|l|}{ Strain } \\
\hline PP47 & crp & Aiba et al. 1982 \\
\hline PP47ssrA & PP47DssrA::Kan & Abo et al. 2000 \\
\hline W3110 & Wild-type & Laboratory stock \\
\hline TA331 & W3110 sssrA::FRT & Abo et al. 2002 \\
\hline TA371 & W3110ssrA $A^{D D_{-} \mathrm{FRT}}$ & Abo et al. 2002 \\
\hline YA002 & $\mathrm{PP} 47 \Delta m r$-FRT & This work \\
\hline YA004 & PP47 $\Delta s s r A:: K a n ~ \Delta m r-F R T$ & This work \\
\hline \multicolumn{3}{|l|}{ Plasmid } \\
\hline $\mathrm{pHA7}$ & $\begin{array}{l}\text { Derivative of pBR322 carrying the crp gene } \\
\text { expressed from the bla promoter }\end{array}$ & Aiba et al. 1982 \\
\hline pHA7M9 & $\begin{array}{l}\text { Derivative of } \mathrm{pHA} 7 \text { in which the } \mathrm{crp} \text { terminator is } \\
\text { mutated by a 2-base substitution in the hairpin } \\
\text { region }\end{array}$ & This work \\
\hline pHA7M6 & $\begin{array}{l}\text { Derivative of pHA7 in which the crp terminator is } \\
\text { extensively mutated }\end{array}$ & Abe et al. 1999 \\
\hline $\mathrm{pHA} 7 \mathrm{~S} 1$ & $\begin{array}{l}\text { Derivative of } \mathrm{pHA} \text { in which the TAA stop codon } \\
\text { of } c r p \text { was changed to AAA }\end{array}$ & Ueda et al. 2002 \\
\hline pYY100 & $\begin{array}{l}\text { Derivative of pBR322 carrying a variant } c r p \\
\text { encoding } \mathrm{His}_{6} \text {-CRP with the wild-type terminator }\end{array}$ & This work \\
\hline pYY101 & $\begin{array}{l}\text { Derivative of pYY101 in which the crp terminator } \\
\text { is mutated by a 2-base substitution in the hairpin } \\
\text { region }\end{array}$ & This work \\
\hline pQE-80L & $\begin{array}{l}\text { Expression vector for } \mathrm{N} \text {-terminally } \mathrm{His}_{6} \text {-tagged } \\
\text { protein }\end{array}$ & QIAGEN \\
\hline pMelR & $\begin{array}{l}\text { Derivative of } \mathrm{pQE}-80 \mathrm{~L} \text { carrying a variant melR } \\
\text { encoding } \mathrm{His}_{6}-\mathrm{MelR}\end{array}$ & This work \\
\hline pSoxR & $\begin{array}{l}\text { Derivative of } \mathrm{pQE}-80 \mathrm{~L} \text { carrying a variant } \operatorname{sox} R \\
\text { encoding } \mathrm{His}_{6}-\mathrm{SoxR}\end{array}$ & This work \\
\hline
\end{tabular}

grown for $20 \mathrm{~min}$ to induce the synthesis of $\mathrm{His}_{6}$-tagged proteins. Total extracts were prepared as described above and analyzed by Western blotting using $6 \times$ His monoclonal antibody (Clontech).

\section{Preparation of total RNA and Northern blot analysis}

Bacterial cells harboring plasmids expressing $\mathrm{CRP}$ or $\mathrm{His}_{6}$-tagged CRP were grown at $37^{\circ} \mathrm{C}$ in $\mathrm{LB}$ medium to mid-log phase, and total RNAs were isolated as described. For the analysis of the stability of crp mRNA, cells harboring pHA7 derivatives were grown in $100 \mathrm{~mL}$ of $\mathrm{LB}$ medium at $37^{\circ} \mathrm{C}$ to mid-log phase, rifampicin was added to $200 \mu \mathrm{g} / \mathrm{mL}$, and incubation was continued. Total RNAs were isolated at time intervals after the addition of rifampicin. For the analysis of melR and soxR mRNAs, cells harboring pMelR and pSoxR were grown at $37^{\circ} \mathrm{C}$ in LB medium to mid-log phase, and the synthesis of melR and soxR mRNAs was induced for $20 \mathrm{~min}$ by adding $0.1 \mathrm{mM}$ IPTG. Total RNAs were isolated.

The total RNAs were resolved by $1.2 \%$ agarose-gel electrophoresis in the presence of formaldehyde and blotted onto Hybond- $\mathrm{N}^{+}$membranes (Amersham) as described (Sambrook et al. 1989). The mRNAs were visualized using digoxigenin (DIG) reagents and kits for nonradioactive nucleic acid labeling and detection system (Roche) according to the procedure specified by the manufacturer. The following DIG-labeled DNA probes were used: 294-bp probe A corresponding to the $5^{\prime}$-crp region; $154-\mathrm{bp}$ probe $\mathrm{B}$ corresponding to the $3^{\prime}$-crp region; 200-bp probe $\mathrm{C}$ corresponding to the $5^{\prime}$-melR region; 200-bp probe $\mathrm{D}$ corresponding to the $5^{\prime}$-soxR region. The DIG-labeled DNA probes were prepared by PCR using DIG-dUTP (Roche).

\section{ACKNOWLEDGMENTS}

We thank Drs. Yoshikazu Nakamura (Tokyo University) and Akira Muto (Hirosaki University) for discussion. This work was supported by Grants-in-Aid from the Ministry of Education, Culture, Sports, Science and Technology of Japan.

The publication costs of this article were defrayed in part by payment of page charges. This article must therefore be hereby marked "advertisement" in accordance with 18 USC section 1734 solely to indicate this fact.

Received November 4, 2002; accepted December 16, 2002.

\section{REFERENCES}

Abe, H. and Aiba, H. 1996. Differential contributions of two elements of $\rho$-independent terminator to transcription termination and mRNA stabilization. Biochimie 78: 1035-1042. 
Abe, H., Abo, T., and Aiba, H. 1999. Regulation of intrinsic terminator by translation in Escherichia coli: Transcription termination at a distance downstream. Genes Cells 4: 87-97.

Abo, T., Inada, T., Ogawa, K., and Aiba, H. 2000. SsrA-mediated tagging and proteolysis of LacI and its role in the regulation of lac operon. EMBO J. 19: 3762-3769.

Abo, T., Ueda, K., Sunohara, T., Ogawa, K., and Aiba, H. 2002. SsrAmediated protein tagging in the presence of miscoding drugs and its physiological role in Escherichia coli. Genes Cells 7: 629-638.

Adhya, S. and Gottesman, M. 1978. Control of transcription termination. Ann. Rev. Biochem. 47: 967-996.

Aiba, H., Fujimoto, S., and Ozaki, N. 1982. Molecular cloning and nucleotide sequencing of the gene for E. coli cAMP receptor protein. Nucleic Acids Res. 10: 1345-1361.

Aiba, H., Hanamura, A., and Yamano, H. 1991. Transcriptional terminator is a positive regulatory element in the expression of the Escherichia coli crp gene. J. Biol. Chem. 266: 1721-1727.

Amabile-Cuevas, C.F. and Demple, B. 1991. Molecular characterization of the soxRS genes of Escherichia coli: Two genes control a superoxide stress regulon. Nucleic Acids Res. 19: 4479-4484.

Carpousis, A.J., Vanzo, N.F., and Raynal, L.C. 1999. mRNA degradation. A tale of poly(A) and multiprotein machines. Trends Genet. 15: $24-28$

Coburn, G.A. and Mackie, G.A. 1999. Degradation of mRNA in Escherichia coli: An old problem with some new twists. Prog. Nucleic Acid Res. Mol. Biol. 62: 55-108.

Collier, J., Binet, E., and Bouloc, P. 2002. Competition between SsrA tagging and translational termination at weak stop codons in Escherichia coli. Mol. Microbiol. 45: 745-754.

Datsenko, K.A., Wanner, B.L. 2000. One-step inactivation of chromosomal genes in Escherichia coli K-12 using PCR products. Proc. Natl. Acad. Sci. 97: 6640-6645.

Frischmeyer, P.A., van Hoof, A., O'Donnell, K., Guerrerio, A.L., Parker, R., and Dietz, H.C. 2002. An mRNA surveillance mechanism that eliminates transcripts lacking termination codons. Science 295: 2258-2261.

Gottesman, S., Roche, E., Zhou, Y., and Sauer, R.T. 1998. The ClpXP and ClpAP proteases degrade proteins with carboxy-terminal peptide tails added by the SsrA-tagging system. Genes \& Dev. 12: 13381347.

Hayes, C.S., Bose, B., and Sauer, R.T. 2002a. Proline residues at the C terminus of nascent chains induce SsrA tagging during translation termination. J. Biol. Chem. 277: 33825-33832.

- 2002b. Stop codons preceded by rare arginine codons are efficient determinants of SsrA tagging in Escherichia coli. Proc. Natl. Acad. Sci. 99: 3440-3445.

Herman, C., Thevenet, D., Bouloc, P., Walker, G.C., and D'Ari, R. 1998. Degradation of carboxy-terminal-tagged cytoplasmic proteins by the Escherichia coli protease HflB (FtsH). Genes \& Dev. 12: $1348-1355$.

Higgins, C.F., Causton, H.C., Dance, G.S.C., and Mudd, E.A. 1993. The role of the $3^{\prime}$ end in mRNA stability and decay. In Control of messenger RNA stability (eds. J. Belasco and G. Brawerman), pp. 13-30. Academic Press, San Diego, CA.

Huang, C., Wolfgang, M.C., Withey, J., Koomey, M., and Friedman, D.I. 2000. Charged tmRNA but not tmRNA-mediated proteolysis is essential for Neisseria gonorrhoeae viability. EMBO J. 19: 1098-1107.

Karzai, A.W. and Sauer, R.T. 2001. Protein factors associated with the SsrA. SmpB tagging and ribosome rescue complex. Proc. Natl.
Acad. Sci. 98: 3040-3044.

Karzai, A.W., Roche, E.D., and Sauer, R.T. 2000. The SsrA-SmpB system for protein tagging, directed degradation and ribosome rescue. Nat. Struct. Biol. 7: 449-455.

Keiler, K.C., Waller, P.R., and Sauer, R.T. 1996. Role of a peptide tagging system in degradation of proteins synthesized from damaged messenger RNA. Science 271: 990-993.

Komine, Y., Kitabatake, M., Yokogawa, T., Nishikawa, K., and Inokuchi, H. 1994. A tRNA-like structure is present in 10Sa RNA, a small stable RNA from Escherichia coli. Proc. Natl. Acad. Sci. 91: 92239227.

Lin-Chao, S., Wei, C.L., and Lin, Y.T. 1999. RNase E is required for the maturation of ssrA RNA and normal ssrA RNA peptide-tagging activity. Proc. Natl. Acad. Sci. 96: 12406-12411.

Maquat, L.E. 2002a. Molecular biology. Skiing toward nonstop mRNA decay. Science 295: 2221-2222.

. 2002b. Nonsense-mediated mRNA decay. Curr. Biol. 12: R196-R197.

Miller, J. 1972. Experiments in molecular genetics. Cold Spring Harbor Laboratory, Cold Spring Harbor, NY.

Regnier, P. and Arraiano, C.M. 2000. Degradation of mRNA in bacteria: Emergence of ubiquitous features. Bioessays 22: 235-244.

Roche, E.D. and Sauer, R.T. 1999. SsrA-mediated peptide tagging caused by rare codons and tRNA scarcity. EMBO J. 18: 4579-4589.

Sambrook, J., Fritsch, E.F., and Maniatis, T. 1989. Molecular cloning: A laboratory manual, 2nd ed. Cold Spring Harbor Laboratory Press, Cold Spring Harbor, NY.

Steege, D.A. 2000. Emerging features of mRNA decay in bacteria. RNA 6: $1079-1090$

Storz, G. 2002. An expanding universe of noncoding RNAs. Science 296: $1260-1263$.

Sunohara, T., Abo, T., Inada, T., and Aiba, H. 2002. The C-terminal amino acid sequence of nascent peptide is a major determinant of SsrA tagging at all three stop codons. RNA 8: 1416-1427.

Tu, G.F., Reid, G.E., Zhang, J.G., Moritz, R.L., and Simpson, R.J. 1995. C-terminal extension of truncated recombinant proteins in Escherichia coli with a 10Sa RNA decapeptide. J. Biol. Chem. 270: 93229326.

Ueda, K., Yamamoto, Y., Ogawa, K., Abo, T., Inokuchi, H., and Aiba, H. 2002. Bacterial SsrA system plays a role in coping with unwanted translational readthrough caused by suppressor tRNAs. Genes Cells 7: 509-519.

Ushida, C., Himeno, H., Watanabe, T., and Muto, A. 1994. tRNA-like structures in 10Sa RNAs of Mycoplasma capricolum and Bacillus subtilis. Nucleic Acids Res. 22: 3392-3396.

van Hoof, A., Frischmeyer, P.A., Dietz, H.C., and Parker, R. 2002. Exosome-mediated recognition and degradation of mRNAs lacking a termination codon. Science 295: 2262-2264.

Wassarman, K.M. 2002. Small RNAs in bacteria: Diverse regulators of gene expression in response to environmental changes. Cell 109: $141-144$

Webster, C., Kempsell, K., Booth, I., and Busby, S. 1987. Organisation of the regulatory region of the Escherichia coli melibiose operon. Gene 59: 253-263.

Withey, J.H. and Friedman D.I. 1999. Analysis of the role of transtranslation in the requirement of tmRNA for $\lambda \mathrm{imm}^{\mathrm{P} 22}$ growth in Escherichia coli. J. Bacteriol. 181: 2148-2157.

. 2002. The biological roles of trans-translation. Curr. Opin. Microbiol. 5: 154-159. 

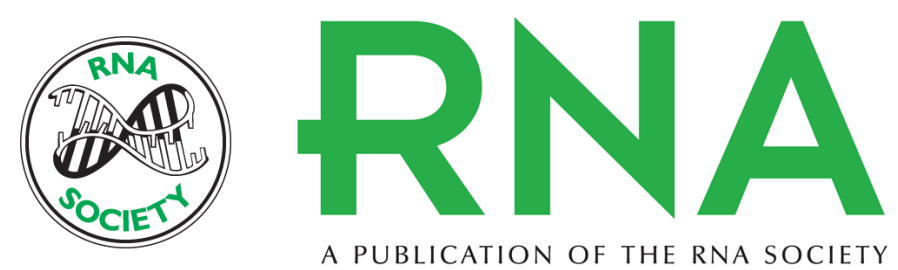

\section{SsrA-mediated trans-translation plays a role in mRNA quality control by facilitating degradation of truncated mRNAs}

YASUFUMI YAMAMOTO, TAKAFUMI SUNOHARA, KAORU JOJIMA, et al.

RNA 2003 9: 408-418

References This article cites 38 articles, 21 of which can be accessed free at: http://rnajournal.cshlp.org/content/9/4/408.full.html\#ref-list-1

License

Email Alerting

Receive free email alerts when new articles cite this article - sign up in the box at the Service top right corner of the article or click here. 